Brit. Heart F., 1966, 28, 350.

\title{
The Wenckebach Phenomenon in Sino-atrial Block
}

\author{
L. SCHAMROTH AND E. DOVE \\ From the Baragwanath Hospital and University of the Witwatersrand, Fohannesburg, South Africa
}

\begin{abstract}
Disturbances in conduction analogous to those occurring at the atrio-ventricular $(\mathrm{A}-\mathrm{V})$ node may be present at the sino-atrial (S-A) junction. When second degree block occurs at the S-A junction, it may adopt the form of the Wenckebach phenomenon. Early descriptions of this arrhythmia were presented by Wenckebach (1906), Rihl (1908), and Wenckebach and Winterberg (1914). There have been relatively few reports since. Barlow (1927), in a review of the subject, found 54 cases, and more recently, Greenwood and Finkelstein (1964), using rigid criteria in their selection, found that earlier reports comprised 219 unequivocal examples of all forms of S-A block of which $38(17 \%)$ were of the Wenckebach type.

We report 4 further cases of S-A block with Wenckebach conduction, which illustrate the diversity of the electrocardiographic presentation.
\end{abstract}

\section{Diagnosis of S-A Wenckebach Conduction}

The sinus impulse is only recorded once it has left the sinus node and activated the atrium-thereby resulting in the $P$ wave. Delay or block in conduction at the S-A junction is, therefore, not visible electrocardiographically, but its presence can be inferred by calculation of certain rhythmic sequences involving the P-P intervals. The principles underlying the Wenckebach form of disturbed S-A conduction are analogous to those governing the Wenckebach phenomenon at the A-V junction.

\section{Principles OF WeNCKeBACH CONDUCTION}

A. $A-V$ Wenckebach Conduction. In the Wenckebach phenomenon at the A-V junction, a progressively increasing conduction delay results in progressively increasing $P-R$ intervals until conduction fails completely and a beat is "dropped". The period of rest afforded to the $A-V$ node by failure of conduction permits its recovery and the sequence begins again. However, each successive

Received June 28, 1965.
$P-R$ interval is increased by an increment which becomes progressively smaller. This is illustrated in Fig. 1 -an idealized representation of the Wenckebach phenomenon. Sinus impulses labelled S1, S2, S3, and S4 are conducted to the ventricles resulting in QRS complexes labelled R1, R2, R3, and R4. Sinus impulse S5 is blocked. The P-R interval becomes progressively longer before the dropped beat. The increment of each P-R interval (shaded area) becomes progressively smaller. The P-P intervals are constant at $60{ }^{\star}$ The first increment, i.e. the increment of the second P-R interval (S2-R2 in this sequence) is 20 thereby resulting in an R-R interval of 80 (R1-R2). The increment of the third P-R interval (S3-R3) is smaller, viz. 10, resulting in an R-R interval of 70 (R2-R3). The increment of the fourth $P-R$ interval is still smaller, viz. 5, resulting in an $R-R$ interval of 65 (R3-R4). Note: Although the P-R interval becomes progressively longer, the increment by which it increases becomes progressively smaller; this results in progressive shortening of the R-R intervals, i.e. the ventricular rate accelerates.

B. S-A Wenckebach Conduction. The idealized representation in Fig. 1 can be applied to S-A Wenckebach conduction by substituting the labels $A, A-V$, and V, with $S$ (sinus node), S-A (sinoatrial junction), and $A$ (atrium)-as indicated in the brackets of the diagram. The ventricular level now becomes the atrial level and the ventricular conduction lines-labelled R1 to R5-become atrial conduction lines and are relabelled P1 to P5 (as indicated in the brackets). Level A, representing the $P$ waves, is the only level visible electrocardiographically. A Wenckebach conduction defect at S-A level will, therefore, present with progressive acceleration of the P-P intervals and is analogous to the progressive acceleration of the $R-R$ intervals in an $\mathrm{A}-\mathrm{V}$ Wenckebach disturbance. This progressive

* All time intervals are expressed in hundredths of a second, i.e. $60=60$ hundredths of a second. 


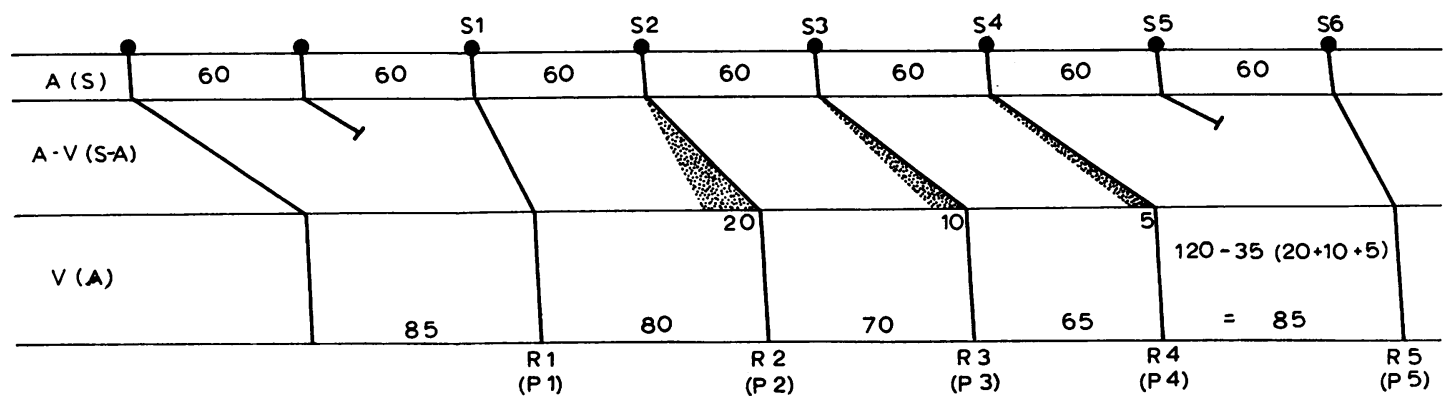

FIG. 1.-Diagram illustrating the principles of Wenckebach conduction. See text.

acceleration of the P-P intervals is followed by an unduly long P-P interval containing the dropped beat.

Note: the S-A interval is the term used to denote the time of conduction from the sinus node to the atria and is analogous to the P-R interval which reflects $\mathrm{A}-\mathrm{V}$ conduction time.

C. Calculation of Sinus Cycle. In A-V Wenckebach conduction the sinus cycle or inter-sinus interval is represented by the P-P interval. In S-A Wenckebach conduction, however, the P-P interval is not an accurate reflection of the sinus interval, as it has been modified by varying S-A conduction times. Nevertheless, the sinus cycle in S-A Wenckebach conduction may be calculated on the basis of certain principles which are evident in Wenckebach conduction at the $\mathrm{A}-\mathrm{V}$ junction. These are considered below.

(1) Sinus cycle in $A-V$ Wenckebach conduction. Although the sinus cycle is evident as the P-P interval in A-V Wenckebach conduction, it may also be calculated independently, i.e. on the basis of the $\mathbf{R}-\mathbf{R}$ intervals only.

In Fig. 1, sinus impulses S1 and S6 both follow a long pause and begin a Wenckebach sequence: they are, therefore, conducted with the same conduction times and have P-R intervals of the same length. Such impulses, which have the same conduction times, will be referred to as corresponding impulses. It follows that the interval between corresponding impulses is maintained through all conduction levels, i.e. the interval S1-S6 must be equivalent to the interval R1-R5. An interval of this type, i.e. one that is the same at adjacent levels will be referred to as an isoconduction interval. As the isoconduction interval S1-S6 contains 5 P-P intervals, the interval R1-R5 must also be equivalent to $5 \mathrm{P}-\mathrm{P}$ intervals. Only $4 \mathrm{R}-\mathrm{R}$ intervals are present between $R 1$ and R5; thus the number of P-P intervals between R1 and R5 is one in excess of the number of $R-R$ intervals. The addition of one extra interval allows for the effect of the single dropped beat. On this basis the sinus cycle can be calculated from the $R-R$ intervals as follows.

(i) The number of R-R intervals within an isoconduction interval is determined. For example, in Fig. 1, there are $4 \mathrm{R}-\mathrm{R}$ intervals within the isoconduction interval R1-R5.

(ii) This number is advanced by one, i.e. to 5 .

(iii) The sinus cycle is obtained by dividing this figure into the isoconduction interval (which measures 300). This corresponds to the following formula :

Formula I: Sinus cycle $=$ Isoconduction interval

No. of sinus cycles within isoconduction interval When there is only one dropped beat, the sinus cycle is equivalent to:

Isoconduction interval

No. of R-R intervals within isoconduction interval +1 Thus in the idealized representation (Fig. 1) the sinus cycle is calculated as follows:

$$
\text { Sinus cycle }=\frac{\mathrm{R} 1-\mathrm{R} 5}{4+1}=\frac{300}{5}=60 .
$$

2. Sinus cycle in $S-A$ Wenckebach conduction. The sinus cycle can be calculated on the basis of Formula I enunciated above. Sinus impulses which are conducted after a long pause will probably have S-A intervals of similar length, i.e. they are corresponding impulses. The isoconduction interval between these corresponding impulses will be the same at both sinus and atrial levels.

Thus when the idealized representation in Fig. 1 is modified for S-A conduction the sinus cycle is calculated as follows:

$$
\text { Sinus cycle }=\frac{P 1-P 5}{4+1}=\frac{300}{5}=60 .
$$

These principles are illustrated in Case 1-a classic form of S-A Wenckebach conduction.

Case 1. The electrocardiogram (Fig. 2) was recorded from a 34-year-old man with mitral stenosis and incompetence who was fully digitalized. An 


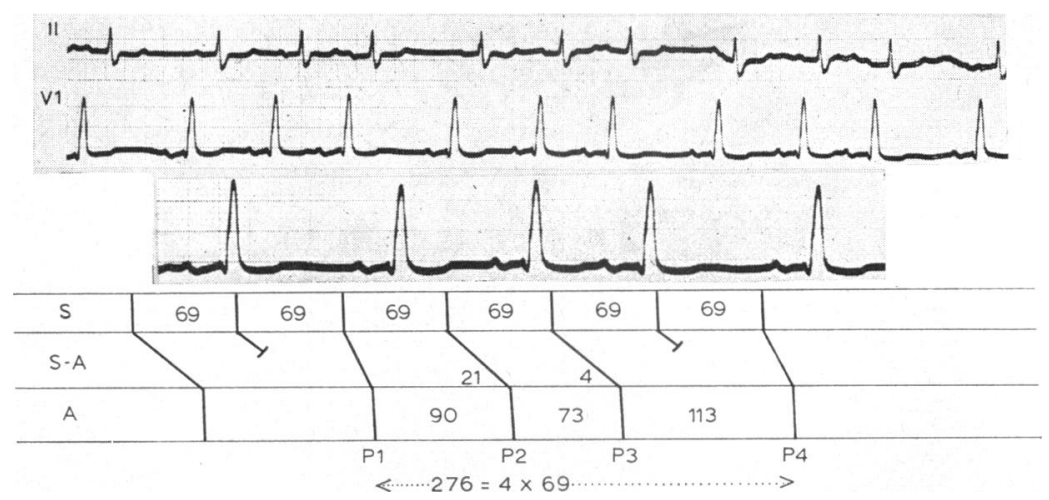

FIG. 2.-Case 1. Electrocardiogram illustrating regular 4:3 S-A block with Wenckebach phenomenon. The middle of the strip recorded by lead VI is enlarged and diagrammed. Note: the A-V nodal and ventricular levels are not diagrammed.

allorhythmia is present consisting of two relatively short and progressively diminishing P-P intervals (measuring 90 and 73, respectively), followed by a long P-P interval of 113. This indicates the presence of an S-A Wenckebach sequence. There is only slight fluctuation of the P-R intervals (20 to 24) and the allorhythmia is thus also reflected in the $\mathbf{R}-\mathbf{R}$ intervals.

Calculation of Sinus Cycle. P1 and P4 each begin a Wenckebach sequence and therefore represent corresponding impulses. The interval P1-P5 is thus an isoconduction interval.

\section{Application of Formula I:}

$$
\text { Sinus cycle }=\frac{\mathrm{P} 1-\mathrm{P} 4}{3+1}=\frac{276}{4}=69 .
$$

The complete Wenckebach sequence is illustrated on the basis of this sinus cycle of 69 (Fig. 2). The first P-P interval (P1-P2) measures 90; the first increment, therefore, measures $21(80-69)$; the second increment measures $4(73-69)$. The total increment equals $25(21+4)$. The long P-P interval of 113 (P3-P4) when corrected for the total increment measures 138; this is equivalent to two sinus cycles of 69 .

Differentiation from Sinus Extrasystoles. An extrasystole may occasionally arise within, or in close proximity to, the S-A node. This gives rise to a premature $\mathbf{P}$ wave whose contour is identical to the $P$ wave recorded by the normal sinus impulse. The following pause is always equal to the normal sinus interval, i.e. it is never compensatory.

In Case 1 the third $P$ wave in each sequence is premature and identical in shape to the other $P$ waves; superficially, therefore, it resembles normal sinus rhythm with a sinus extrasystole after every two sinus impulses. This diagnosis, however, is not substantiated by the P-P intervals, as the pause following each extrasystole should be equal to the sinus cycle preceding it, i.e. the interval P3-P4 (113) should be equal to the interval P1 - P2 (90).

The aforementioned principles are easily applicable in the classic S-A Wenckebach phenomenon, i.e. when only one beat is dropped before the sequence begins again. When a Wenckebach sequence is followed by two or more successively dropped beats the situation becomes more complex as the exact number of sinus cycles within an isoconduction interval is not immediately apparent. This is illustrated in Cases 2, 3, and 4.

Case 2. The electrocardiogram (Fig. 3) was recorded from a 46-year-old woman with hypertensive cardiac failure and uræmia; she was fully digitalized. The electrocardiogram (standard lead II) shows a group of five PQRS complexes, which is preceded and followed by a long pause. There is progressive diminution of the P-P intervals within this group indicating a Wenckebach conduction sequence at the S-A junction. Note: the QRS complexes are all preceded by a P-R interval of the same length; the arrhythmia, therefore, affects the QRS complexes to the same degree as the $P$ waves, and thus there is also a progressive diminution of the R-R intervals.

It is evident from the idealized representation of the Wenckebach phenomenon (Fig. 1) that the shortest interval still bears an increment; the sinus cycle must, therefore, be shorter than the shortest $\mathbf{P}-\mathbf{P}$ interval. Thus the shortest P-P interval in Fig. 1 measures 65 whereas the true sinus cycle measures 60 . Furthermore, since the last increment of a sequence is the smallest, it follows that the last P-P interval in a Wenckebach sequence will 


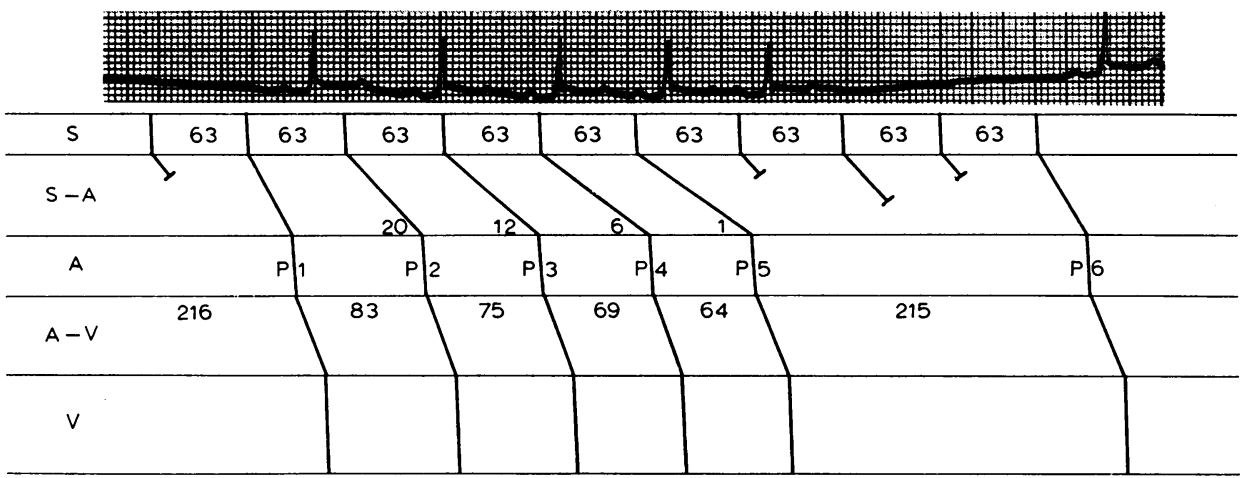

FIG. 3.-Case 2. Electrocardiogram illustrating S-A Wenckebach conduction followed by a period of $4: 1$ S-A block.

be the one that is most closely approximate to the sinus cycle.

Based on the above-mentioned principle, the sinus cycle in the tracing shown in Fig. 3 must approximate, but be less than, the terminal P-P interval (interval P4-P5) which measures 64. It follows that the succeeding long interval of 215 must contain more than one dropped beat. The exact number of sinus cycles within the isoconduction interval P1-P6 is thus not apparent. Furthermore, Formula I can only be applied once the exact number of sinus cycles (including all dropped beats within an isoconduction interval) is known. The exact number of sinus cycles in these circumstances can be calculated on the following basis:

1. The interval most approximate to the sinus cycle is the last and shortest P-P interval in a sequence.

2. This interval is divided into the isoconduction interval. The result will approximate the number of sinus cycles within the isoconduction interval. However, as this terminal P-P interval is slightly longer than the true sinus cycle, the answer will of necessity be less than the number of sinus cycles contained within this isoconduction interval and must therefore be corrected to the next whole number. Thus the formula for calculation of the number of sinus cycles within an isoconduction interval may be expressed as follows.

\section{Formula II:}

No. of sinus cycles within isoconduction interval $=$ isoconduction interval shortest P-P interval - corrected to next whole number.

Once the number of sinus cycles within an isoconduction is known, the exact duration of the sinus cycle may be obtained by application of Formula I.

These principles are applied to Case 2 (Fig. 3) as follows:
1. P1 and P6 represent corresponding impulses. The interval P1-P6 is, therefore, by definition an isoconduction interval.

2. The shortest $\mathbf{P}-\mathbf{P}$ interval is interval $\mathbf{P 4 - P 5}$ and measures 64.

Application of Formula II: Number of sinus cycles within the interval $\mathrm{P} 2-\mathrm{P} 7=\frac{\mathrm{P} 1-\mathrm{P} 6}{\mathrm{P} 4-\mathrm{P} 5}$ corrected to next whole number $=\frac{503}{64}=7 \cdot 8$ corrected to 8 . There are thus 8 sinus cycles between P1 and P6.

Application of Formula I: Sinus cycle = isoconduction interval

No. of sinus cycles within isoconduction interval $=\frac{503}{8}=62 \cdot 9$ or 63 .

On the basis of a sinus cycle of 63 , the Wenckebach sequence in this group is represented as follows (Fig. 3). The first increment is $20(83-63)$, the second increment $12(75-63)$, the third increment $6(69-63)$, and the fourth increment $1(64-63)$. The sum of all the increments measures 39 $(20+12+6+1)$. The long P-P interval P5-P6 measures 215; when this figure is corrected for the total increment it measures $254(215+39)$. This is equivalent to four sinus intervals of 63.5 .

Case 3. The electrocardiogram (Fig. 4) was recorded from a 56-year-old Bantu man with cryptogenic cardiomyopathy-a form of congestive cardiomyopathy commonly found in the Bantu (negro) race of South Africa. He was on digitalis therapy. The electrocardiographic strip labelled I (standard lead II) was recorded (as a control tracing) before the intravenous administration of a betareceptor blocking agent-propranolol (inderal). It shows normal sinus rhythm with a P-P interval of 


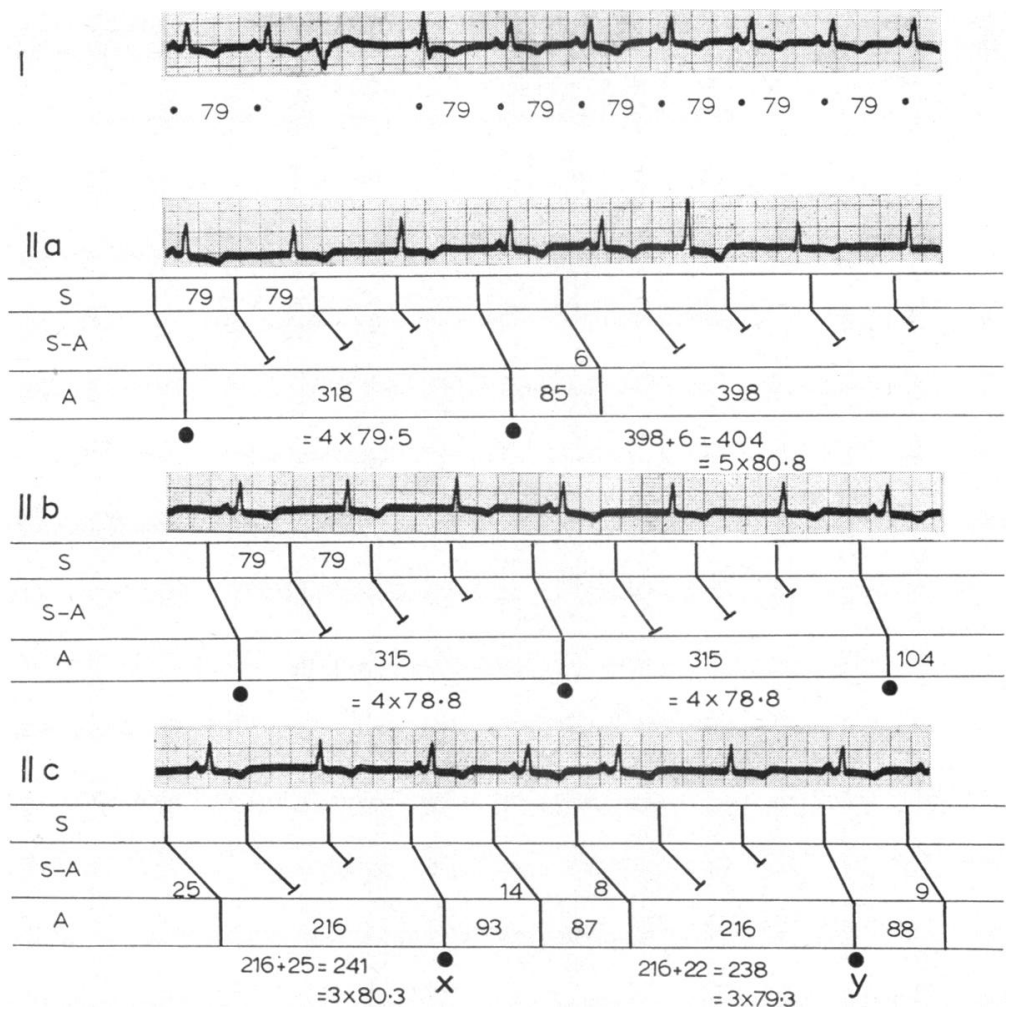

Fig. 4.-Case 3. Strip 1: control tracing (standard lead II) recorded before the administration of propranolol. Strip II: continuous recording following the administration of propranolol, illustrating highgrade S-A block and several Wenckebach sequences. See text. Note: the A-V nodal and ventricular levels are not diagrammed.

79. There is also one ventricular extrasystole followed by an A-V nodal escape beat which is dissociated from the near-coincident $P$ wave. Strip II ( $a, b$, and $c$ form a continuous recording of standard lead II) was recorded 5 minutes after the administration of propranolol and shows A-V nodal escape rhythm consequent to marked sinus depression. The P-P intervals are markedly and irregularly prolonged, resulting in an apparent haphazard distribution of $P$ waves with varying P-P intervals. In row IIc, three $P$ waves follow each other in close succession and show progressive diminution of the P-P intervals, viz. 93 and 87 . This is followed by a long P-P interval which measures 216. This sequence suggests the presence of a S-A Wenckebach phenomenon and is the only indication of this arrhythmia in the entire recording.

Applying the aforementioned formulæ, the sinus cycle may be calculated as follows.

The $P$ waves labelled $X$ and $Y$ in row IIc follow long P-P intervals and initiate conduction sequences. They are, therefore, conducted with the same S-A intervals and represent corresponding impulses.
Consequently, the interval $\mathrm{X}-\mathrm{Y}$ is, by definition, an isoconduction interval and measures 396 $(93+87+216)$.

Application of Formula II: Number of sinus cycles within the isoconduction interval $\mathrm{X}-\mathrm{Y}=$

$$
\frac{396}{87}=4.5 \text { corrected to } 5 \text {. }
$$

Application of Formula I: Sinus cycle $=\frac{396}{5}=79 \cdot 2$. Confirmatory evidence for this sinus cycle of 79 is present in the control tracing which also reveals the same sinus cycle of 79 .

Furthermore, since the shortest P-P interval measures 87 , and the long pause that follows it measures 216 , it is clear that there must be more than one dropped beat within the interval of 216 .

Note: there are other isoconduction intervals and corresponding impulses (labelled with black dots) in this tracing. These intervals when used in the above formulæ also reveal a sinus cycle of 79 .

The complete Wenckebach sequence is represented on the basis of the sinus cycle of 79 (Fig. 4 
(IIc)). The first increment in the sequence $X-Y$ is $14(93-79)$, the second $8(87-79)$, and the long pause is equivalent to 3 sinus cycles, i.e. the long pause + the total increment $=238(216+22)$; this is equivalent to three sinus cycles of $79 \cdot 3$.

Case 4. The electrocardiogram (Fig. 5) was recorded from a woman aged 28 years with mitral incompetence and stenosis, who was fully digitalized. The tracing shows considerable variation of the P-P intervals which, at first glance, appear to reflect a marked sinus arrhythmia. There are, however, numerous sequences where the P-P intervals become progressively shorter followed by a sudden long pause; this suggests the presence of an S-A Wenckebach phenomenon. For example, an allorhythmia is present in the upper strip consisting of two progressively diminishing P-P intervals followed by a long pause. Similar sequences are seen scattered throughout the tracing. A long Wenckebach sequence is seen at the beginning of the third strip (diagrammed) in which successive P-P intervals measure $81,67,62,59$, and 59 , respectively. This sequence is followed by a long pause of 105 . The first and seventh $P$ waves in this strip represent corresponding impulses and the isoconduction interval between them measures 433 . The shortest P-P interval within this sequence measures 59.
Calculation of Sinus Cycle

Application of Formula II: The number of sinus cycles within the aforementioned isoconduction interval is: $\frac{433}{59}=7 \cdot 3$ corrected to 8 .

Application of Formula I: The true sinus cycle measures $\frac{433}{8}=54$.

The complete sequence is diagrammed on this basis. Successive increments measure $27,13,8,5$, and 5 , the total being 58. The long P-P interval of 105 when corrected for the total increment measures $163(105+58)$ and is equivalent to three sinus intervals of 54 .

This Wenckebach sequence is followed by relatively regular rhythm with P-P intervals measuring $105,104,98,102,100,101,109,110$, and 104 . On the basis of the calculated sinus cycle of \pm 54 , these intervals represent sinus rhythm with a 2:1 S-A block; the rhythm is complicated by a slight sinus arrhythmia.

\section{DifFERENTIATION OF S-A WeNCKebach CONDUCTION FROM SINUS ARRHYTHMIA}

In sinus arrhythmia, the gradual shortening of

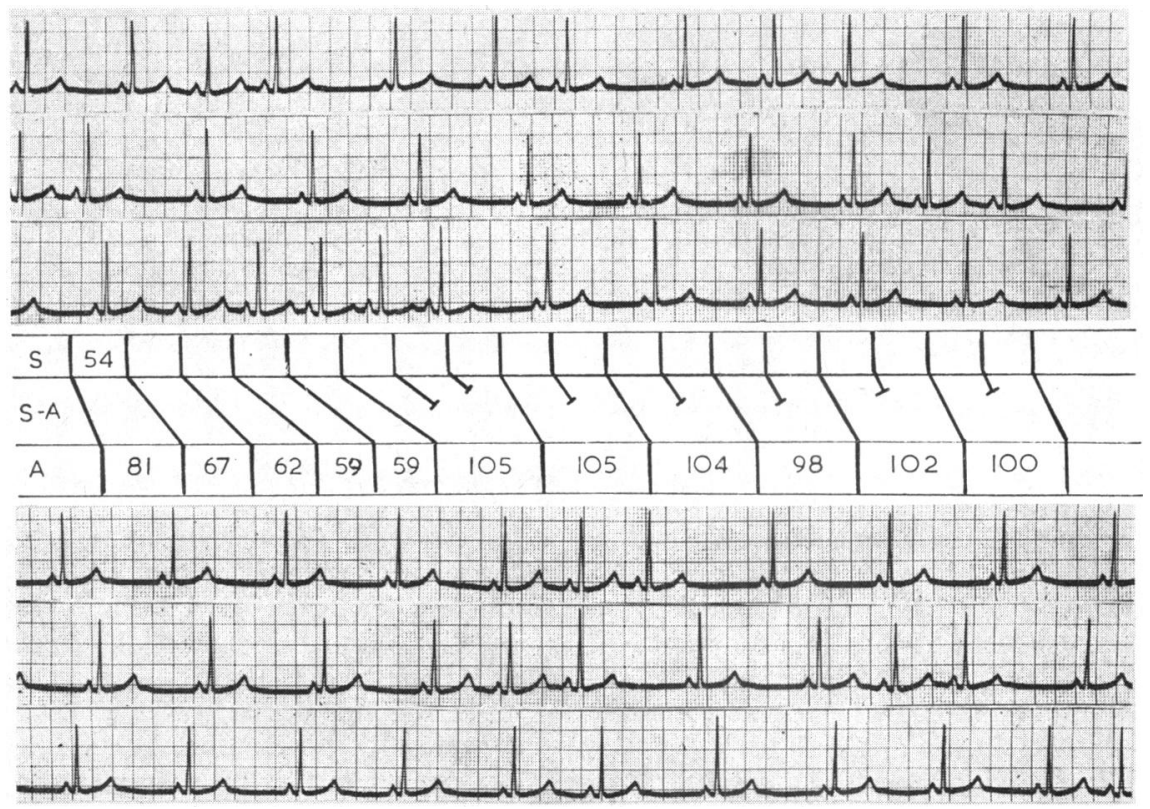

FIG. 5.-Case 4. Electrocardiogram (continuous strip of standard lead II) illustrating marked sinus irregularity. Numerous Wenckebach sequences are interspersed with periods of 2:1 S-A block. See text. The diagram refers to the third strip only. Note: the A-V nodal and ventricular levels are not diagrammed. The effect of plotting each P-P interval against its succeeding P-P interval is illustrated in Diagram C of Fig. 6. 

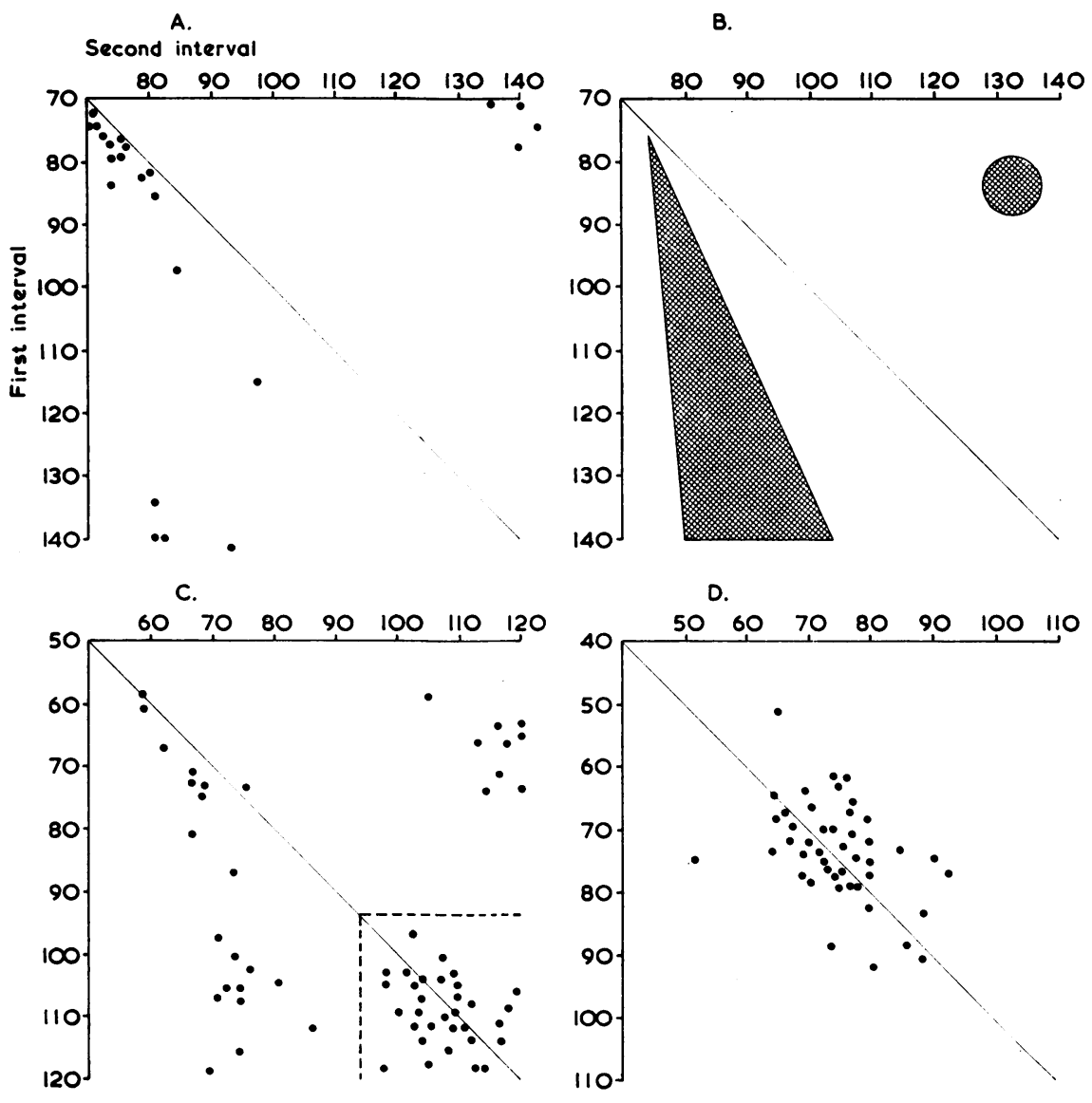

FIG. 6.-Graphic illustrations of the effect of plotting each P-P or R-R interval against its succeeding interval. See text. (A) The distributional pattern with A-V Wenckebach conduction. (B) Diagrammatic representation of the classic Wenckebach distribution. (C) The distributional pattern of S-A Wenckebach conduction; graphed from the tracing shown in Fig. 5. (D) The distributional pattern of the sinus arrhythmia depicted in Fig. 7.

$\mathrm{P}-\mathrm{P}$ intervals is followed by a gradual lengthening. In contrast to this, the gradual shortening in an S-A block with Wenckebach conduction is followed by a sudden long pause which is again followed by gradual shortening. The distinction between the two arrhythmias is not always apparent, especially when S-A Wenckebach block is complicated by sinus arrhythmia and/or a regular Mobitz type S-A block, e.g. $2: 1$ or 3:1 S-A block with fixed S-A interval. The differentiation of these arrhythmias is facilitated by the following procedure.

Each P-P interval is plotted against its succeeding $\mathbf{P}-\mathbf{P}$ interval. The first $\mathbf{P}-\mathbf{P}$ interval of each pair is plotted on a descending $Y$ axis, the second on the $\mathrm{X}$ axis (Fig. 6). Every point thus represents a pair of intervals. A diagonal line-the leading diagonal -is drawn through all points with equal co-ordin- ates. The distribution of the co-ordinates in relation to this leading diagonal reveals characteristic graphic patterns for sinus arrhythmia and S-A Wenckebach conduction.

In sinus arrhythmia, the gradual lengthening and subsequent shortening of succeeding P-P intervals results in an even distribution-with uniform population - on either side of the leading diagonal. This is illustrated in Fig. 6D-a graph reflecting the sinus arrhythmia depicted in Fig. 7.

A typical distribution of a Wenckebach sequence is evident when this procedure is used with $A-V$ Wenckebach conduction (in this instance, each R-R interval is plotted against its succeeding $R-R$ interval). Fig. 6A reflects the pattern of an $A-V$ Wenckebach block (electrocardiogram not illustrated). The gradual shortening followed by an 


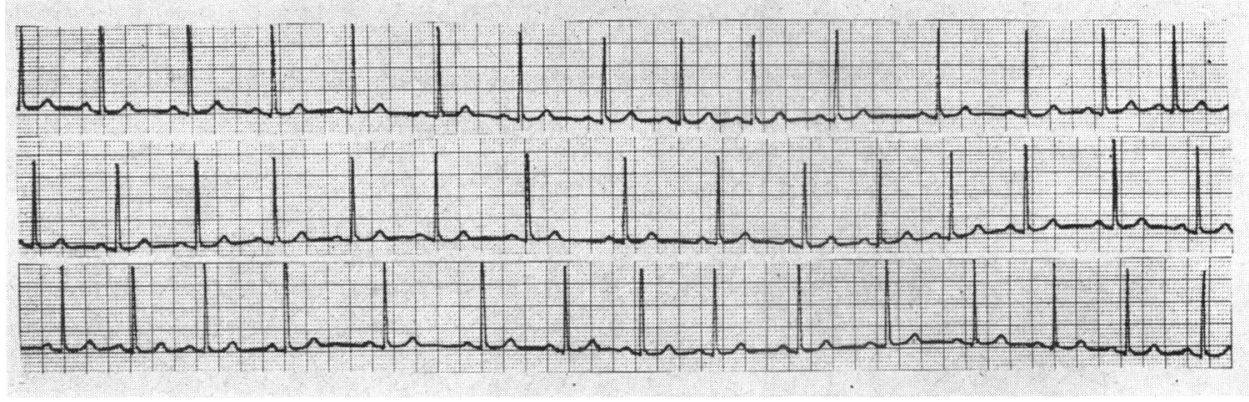

FIG. 7.-Electrocardiogram (continuous strip of standard lead II) illustrating sinus arrhythmia. The effect of plotting each P-P interval against its succeeding P-P interval is illustrated in Fig. 6D.

abrupt long pause results in co-ordinates having the following characteristic distributional pattern: the points are concentrated in two areas.

1. A large concentration on the lower side of the leading diagonal with a higher density towards the upper region.

2. A small cluster of low density in the right upper corner-above the leading diagonal.

Note: There are no points in the region immediately above the leading diagonal.

Fig. 6B is an idealized diagrammatic representation of this distribution-the shaded areas represent the regions of concentration.

This characteristic distribution is revealed in Fig. 6 when the method is applied to Case 4. A small cluster of points is located in the top righthand corner above the leading diagonal; a large concentration occurs below the leading diagonal. In addition, a third concentration is present around the lower end of the leading diagonal (area enclosed by the dotted lines); this concentration is evenly distributed on either side of the leading diagonal and reflects the periods of 2:1 S-A block-complicated by slight sinus arrhythmia. Note: Outside the area enclosed by the dotted lines, there are virtually no points in the region immediately above the leading diagonal.

\section{Discussion}

Winton (1948) presented three criteria for the diagnosis of S-A Wenckebach conduction.

(1) The P-P interval including a blocked S-A impulse is shorter than double the distance of the $\mathrm{P}-\mathrm{P}$ interval preceding it.

(2) The P-P interval following the dropped S-A impulse is larger than the interval preceding it.

(3) There is a progressive diminution of the P-P intervals.

The second and third of Winton's criteria can be applied to all our cases. The P-P interval following the long pause is always larger than the interval preceding it, and in each example there is a progressive diminution of the P-P intervals. This progressive diminution is evident in but one short sequence in Case 3 and provides the only clue to the presence of a S-A Wenckebach phenomenon.

The first of Winton's criteria is valid if there is only one dropped beat. Whereas a single dropped beat is usual with an $\mathrm{A}-\mathrm{V}$ Wenckebach conduction sequence, in an S-A Wenckebach phenomenon the long pause frequently contains two or more successive dropped beats. Thus Winton's first criterion does not apply to our 2nd, 3rd, and 4th cases where the long pause following a Wenckebach sequence contains more than one consecutively dropped beat. In Case 2 the long pause represents a 4:1 S-A block; in Case 3 the long pauses represent $3: 1,4: 1$, and 5:1 S-A block; and in Case 4 the long pauses represent 2:1 and 3:1 S-A block.

Greenwood and Finkelstein (1964) have modified Winton's first criterion to read "the (long) pause is less than a multiple of the basic sinus rate". This, however, is only applicable once the basic sinus cycle is known. Thus calculation of the sinus cycle (as revealed by the aforementioned formulæ) is an essential prerequisite to the elucidation of an S-A Wenckebach structure.

S-A Wenckebach conduction is usually associated with heart disease. In the 38 cases reviewed by Greenwood and Finkelstein (1964), most examples were associated with arteriosclerotic heart disease (13) and hypertensive heart disease (6); a few cases were associated with rheumatic heart disease (4) and coronary heart disease (3); isolated examples were found with diphtheria, tetany, myocarditis, influenza, and syphilitic and congenital heart disease. Five cases were not associated with heart disease. All our patients had advanced heart disease and were on digitalis therapy. In Case 3, however, the 
arrhythmia was clearly precipitated by the intravenous injection of propranolol (inderal).

S-A Wenckebach conduction is thus more likely to be associated with a diseased heart and its recognition and distinction from the benign sinus arrhythmia is of importance. The arrhythmia is probably not as uncommon as the paucity of reports would suggest. More frequent diagnosis will result from longer recordings and an increased awareness of the classical sign, viz. progressive diminution of P-P intervals. Calculation should then be instituted to confirm the presence of an S-A Wenckebach phenomenon.

\section{SUMMARY}

Four cases are presented illustrating the diversity of the electrocardiographic presentation of sinoatrial Wenckebach conduction. All the patients had advanced heart disease and were receiving digitalis therapy. In one case the arrhythmia was precipitated by the intravenous injection of propranolol (inderal).

The principles governing the arrhythmia are described and formulæ are presented for the calculation of the true sinus cycle. Previous diagnostic criteria for this arrhythmia are critically reviewed.
Sino-atrial Wenckebach conduction may masquarade as conspicuous sinus arrhythmia, and is not as uncommon as the paucity of published reports would suggest. A method of distinguishing sinoatrial- Wenckebach conduction from the relatively benign sinus arrhythmia is described.

This study was assisted by a grant to one of us (L.S.) from the South African Council for Scientific and Industrial Research; the assistance is acknowledged with thanks. We wish to thank the Photographic Unit, Department of Medicine, University of the Witwatersrand, for the photographic reproductions.

\section{REFERENCES}

Barlow, P. (1927). The clinical occurrence of sinoauricular block. Lancet, 1, 65.

Greenwood, R. J., and Finkelstein, D. (1964). Sinoatrial Heart Block. Charles C. Thomas, Springfield, Illinois.

Rihl, J. (1908). Klinischer Beitrag zur Kenntnis der Utberleitungsstörungen von der Bildungsstätte der Ursprungsreize zum Vorhof. Dtsch. Arch. klin. Med., 94, 286.

Wenckebach, K. F. (1906). Beiträge zur Kenntnis der menschlichen Herztätigkeit. Arch. Anat. Physiol., [Physiol. Abt.] (Lpz.), 297.

Herztätigkeit und ihre klinische Bedeutung, p. 75. Engelmann, Leipzig.

Winton, S. S. (1948). Sino-auricular block: An analysis of eleven cases. Acta cardiol. (Brux.), 3, 108. 